\title{
Triple Higgs boson production at the ILC within a generic Two-Higgs-Doublet Model
}

\author{
Giancarlo Ferrera* \\ Dipartimento di Fisica, Università di Firenze \& INFN Sezione di Firenze \\ Via G. Sansone 1, I-50019 Sesto Fiorentino, Florence, Italy \\ E-mail: ferreralfi.infn.it \\ Jaume Guasch \\ Gravitation and Cosmology Group, Dept. FF \& Institut de Ciències del Cosmos, \\ Universitat de Barcelona \\ Av. Diagonal 647, E-08028 Barcelona, Catalonia, Spain \\ E-mail: jaume.guasch@ub.edu

\section{David López-Val} \\ High Energy Physics Group, Dept. ECM, \\ Universitat de Barcelona \\ Av. Diagonal 647, E-08028 Barcelona, Catalonia, Spain \\ E-mail: dlopez@ecm.ub.es

\section{Joan Solà} \\ High Energy Physics Group, Dept. ECM \& Institut de Ciències del Cosmos, \\ Universitat de Barcelona \\ Av. Diagonal 647, E-08028 Barcelona, Catalonia, Spain \\ E-mail: solaeifae.es
}

\begin{abstract}
We present a study of triple Higgs boson $(3 \mathrm{H})$ production at the International Linear Collider (ILC) within the general Two-Higgs-Doublet Model (2HDM). We compute the production crosssections at the leading-order for the $3 \mathrm{H}$ final states and find values up to $\sigma \sim 0.1 \mathrm{pb}$. This result represents a large enhancement with respect to the corresponding MSSM cross-sections, which stay typically at the level of $\sigma \sim 10^{-6} \mathrm{pb}$ or less. Furthermore, since the $3 \mathrm{H}$ cross-sections in the general $2 \mathrm{HDM}$ can be of the order of the double Higgs production cross-sections, such $3 \mathrm{H}$ processes could be a competitive (if not the dominant) mechanism for Higgs boson production at the ILC. In practice, these $3 \mathrm{H}$ events could be identified through the tagging of 6 heavy-quark jet final states and, in this case, they would provide strong evidence of an extended Higgs boson sector - likely of non-supersymmetric nature.
\end{abstract}

8th International Symposium on Radiative Corrections

October 1-5, 2007

Florence, Italy

${ }^{*}$ Speaker. 


\section{Introduction}

Double Higgs boson (2H) production in a linear collider $e^{+} e^{-} \rightarrow h^{0} A^{0} ; H^{0} A^{0} ; H^{+} H^{-}$, has been investigated in great detail in the literature, although mainly in the Minimal Supersymmetric Standard Model (MSSM) [1]. However, a tree-level analysis of these pairwise-produced unconventional Higgs bosons is most likely insufficient to disclose their true nature. It is for this reason that a dedicated work on radiative correction calculations on Higgs boson production has been undertaken in various models $[2,3]$. Besides, a second interesting mechanism for studying the Higgs boson properties is the triple Higgs $(3 \mathrm{H})$ production, which carries essential information to trace back the ultimate structure of the corresponding Higgs potential. This kind of processes have been profusely studied also within the MSSM, although in this case the cross-sections are rather meager [4]. Our main purpose here is to study the trilinear coupling $H H H$ in the general Two-Higgs-Doublet Model (2HDM) by focusing on the $3 \mathrm{H}$ final states produced at the ILC [5]:

$$
e^{+} e^{-} \rightarrow H^{+} H^{-} h_{i}, \quad e^{+} e^{-} \rightarrow h_{i} h_{i} A^{0}, \quad e^{+} e^{-} \rightarrow h^{0} H^{0} A^{0}, \quad\left(h_{i}=h^{0}, H^{0}, A^{0}\right) .
$$

Let us recall that the general 2HDM [6] is obtained by canonically extending the SM Higgs sector with a second $S U(2)_{L}$ doublet with weak hypercharge $Y=1$, so that it contains 4 complex scalar fields. The free parameters $\lambda_{i}$ in the general, CP-conserving, 2HDM potential can be finally expressed in terms of the masses of the physical Higgs particles $\left(M_{h^{0}}, M_{H^{0}}, M_{A^{0}}, M_{H^{ \pm}}\right), \tan \beta$ (the ratio of the two VEV's $\left\langle H_{i}^{0}\right\rangle$ giving masses to the up- and down-like quarks) and the mixing angle $\alpha$ between the two $C P$-even states. There remains, however, the coupling $\lambda_{5}$, which cannot be absorbed in the previous quantities. Following [7], we set $\lambda_{5}=\lambda_{6}=2 \sqrt{2} G_{F} M_{A^{0}}^{2}$. This condition allows to keep closer to the MSSM structure of the Higgs sector. Therefore we end up with 6 free parameters, to wit: $\left(M_{h^{0}}, M_{H^{0}}, M_{A^{0}}, M_{H^{ \pm}}, \tan \alpha, \tan \beta\right)$. Furthermore, to ensure the absence of treelevel flavor changing neutral currents (FCNC), two main 2HDM scenarios arise: 1) type I 2HDM, in which one Higgs doublet couples only to down-like quarks, whereas the other doublet does not couple to any quark; 2) type II $2 \mathrm{HDM}$, where one doublet couples only to down-like quarks and the other doublet to up-like quarks. The MSSM Higgs sector is actually a type II one, but of a very restricted sort (enforced by SUSY invariance) [6].

Further constraints must be imposed to attest that the SM behavior is sufficiently well reproduced up to the energies explored so far, namely: $i$ ) the perturbativity and unitarity bounds; $i i$ ) the approximate $S U(2)$ custodial symmetry, i.e. $\left|\delta \rho_{2 H D M}\right| \leq 10^{-3}$ [8], and iii) consistency with the low-energy radiative $B$-meson decay (which entails $M_{H^{ \pm}}>350 \mathrm{GeV}$ for $\tan \beta \geq 1$ [9], unless we consider type-I 2HDM). We refer the reader to [5] for further details, in particular for the full list of trilinear couplings within the general 2HDM that are relevant for the present calculation.

\section{Double and triple Higgs boson production in the 2HDM}

In what follows we shall mainly discuss the leading-order $3 \mathrm{H}$ production at the ILC within the general $2 \mathrm{HDM}$ and compare briefly with the $2 \mathrm{H}$ production processes. Throughout the present work, we have made used of the standard computational packages [10].

We begin by reporting on the tree-level results for the $2 \mathrm{H}$ production within the $2 \mathrm{HDM}$, specifically on the CP-conserving channels $e^{+} e^{-} \rightarrow h^{0} A^{0} ; H^{0} A^{0} ; H^{+} H^{-}$. Let us firstly define two diffe- 
rent scenarios: (a) light, and (b) heavy, Higgs boson masses (see Table 1). We wish to use set I and III for the study of $2 \mathrm{H}$ production, while set II and set III for the study of $3 \mathrm{H}$ production.

\begin{tabular}{|c||c|c|c|}
\hline & Set I & Set II & Set III \\
\hline \hline$M_{h^{0}}(\mathrm{GeV})$ & 100 & 100 & 200 \\
\hline$M_{H^{ \pm}}(\mathrm{GeV})$ & 120 & 120 & 350 \\
\hline$M_{H^{0}}(\mathrm{GeV})$ & 150 & 150 & 250 \\
\hline$M_{A^{0}}(\mathrm{GeV})$ & 140 & 300 & 340 \\
\hline
\end{tabular}

Table 1: Sets I, II and III of light and heavy Higgs boson masses in the 2HDM. Sets I and III are used for $2 \mathrm{H}$ production and Sets II and III for $3 \mathrm{H}$ production.

We have found [5] that, in the light Higgs boson mass regime, the $2 \mathrm{H}$ production rates within the 2HDM are substantial (a few thousands events per $100 \mathrm{fb}^{-1}$ of integrated luminosity), with maximum values reaching $\sigma\left(e^{+} e^{-} \rightarrow H^{+} H^{-}\right) \sim 0.1 \mathrm{pb}$, whereas for heavier Higgs masses (Set III) the optimal production rates lie around one order of magnitude below. Nevertheless, even in these less favored scenarios, the predicted rates are still quite sizeable within the clean ILC environment. Worth noting is that the predicted cross-sections for the same $2 \mathrm{H}$ processes within the MSSM yield $\sigma \sim 10^{-2} \mathrm{pb}$ and are thus comparable to the $2 \mathrm{HDM}$ values for similar masses. This is a reflex of the fact that formally the Higgs-Higgs-gauge boson couplings do not differ from one model to the other. Therefore, we conclude that sizeable rates of non-standard $2 \mathrm{H}$ production can be achieved at the ILC for both SUSY and non-SUSY extended Higgs sectors, which implies that both scenarios are hard to distinguish at the tree-level. A clear separation of them can only be accomplished through the detailed study of radiative corrections to $2 \mathrm{H}$ production in both the MSSM [2] and the 2HDM [3].

Let us now discuss the case of the triple Higgs boson production (cf. Eq. (1.1)) within the general 2HDM (see Ref. [5] for further details and discussions). The basic result here is very different from the $2 \mathrm{H}$ case sketched above, in the sense that the $3 \mathrm{H}$ cross-sections for the general $2 \mathrm{HDM}$ may carry large enhancements at the tree-level which are not present in the MSSM. It means that the $3 \mathrm{H}$ channels in the general $2 \mathrm{HDM}$ could be, in contrast to the $2 \mathrm{H}$ ones, truly distinctive already at the leading-order. The dynamical reason for this stems from the structure of the trilinear Higgs boson couplings $\mathrm{HHH}$ in the general 2HDM. In contrast to the Yukawa coupling with fermions, these trilinear couplings do not depend on whether we are in type I or type II models, and can be largely enhanced in certain regions of the parameter space (see Table 1 of [5]). Such enhancements are not possible in the MSSM case, owing to the purely gauge nature of these couplings in the SUSY case.

The numerical analysis fully corroborates our expectations. In Fig. 1 we have plotted the $3 \mathrm{H}$ production cross-sections within the 2HDM. Again, two different mass regimes (sets II and III in Table 1) are considered. This is so because of the constraints imposed by the radiative $B$-meson decays on the charged Higgs boson masses for type II models $\left(M_{H^{ \pm}} \gtrsim 350 \mathrm{GeV}\right)$ [9]. Lighter Higgs boson regimes (allowed for type I models) entail optimal cross-sections at the level of $\sim 0.1$ $\mathrm{pb}$ or more, therefore implying promising rates of at least $10^{4}$ events per $100 \mathrm{fb}^{-1}$ of integrated luminosity. The corresponding results for the heavy Higgs boson scenario lie around $1-2$ orders of magnitude below those for the light Higgs boson scenario, and they are attained at higher values of $\sqrt{s}$. The results for set III (suited for type II models) translate into rates of $O\left(10^{2}-10^{3}\right)$ events 
per $100 \mathrm{fb}^{-1}$ of integrated luminosity, which may still allow some comfortable detection of the signal in the clean ILC environment. The remaining $3 \mathrm{H}$ channels (among the $7 \mathrm{CP}$-conserving triple final states in (1.1)) provide smaller production rates in optimal conditions [5].
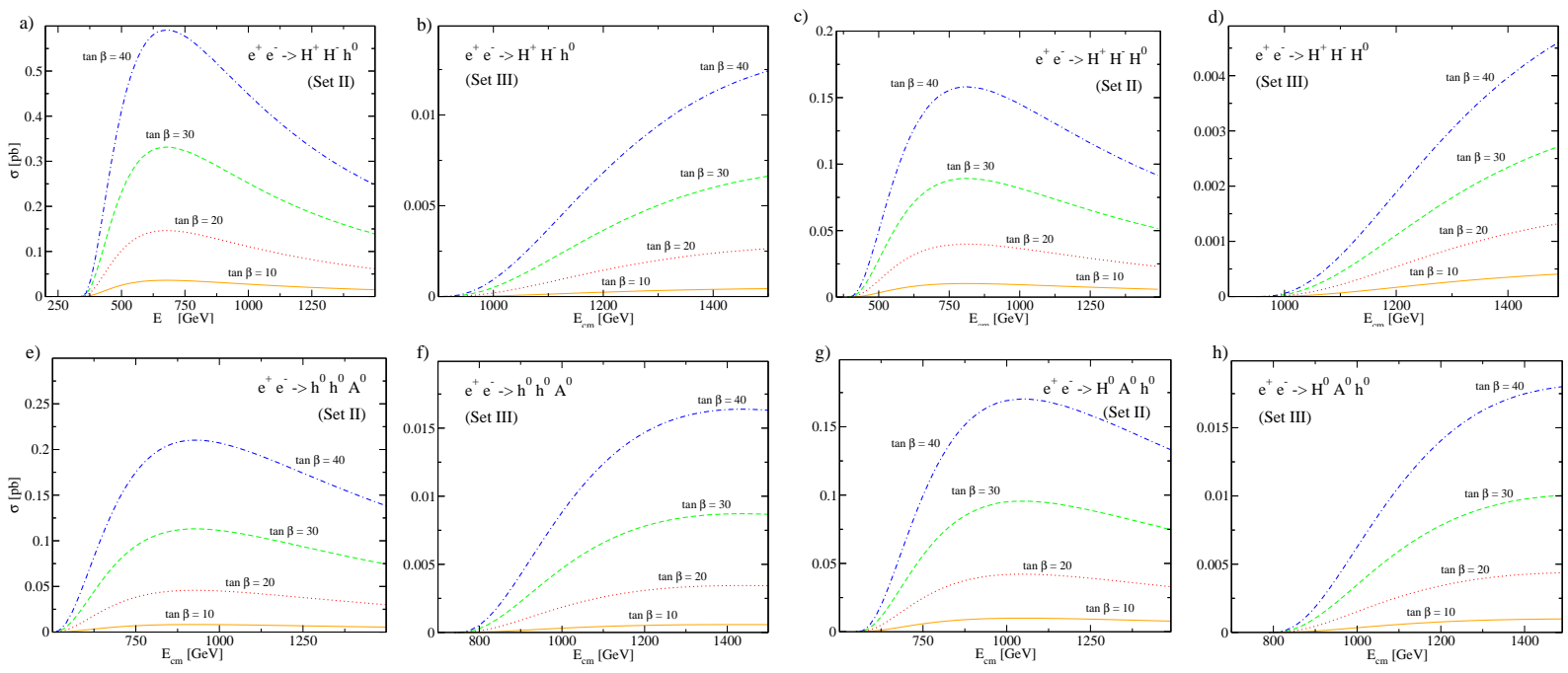

Figure 1: Total cross-section $\sigma(\mathrm{pb})$ for the triple Higgs boson production processes $e^{+} e^{-} \rightarrow H^{+} H^{-} h^{0}, e^{+} e^{-} \rightarrow$ $H^{+} H^{-} H^{0}, e^{+} e^{-} \rightarrow h^{0} h^{0} A^{0}$ and $e^{+} e^{-} \rightarrow H^{0} A^{0} h^{0}$ in the general 2HDM as a function of $\sqrt{s}$ and for different values of $\tan \beta$. In each case the label of the process and the choice (Set II or Set III) of Higgs boson masses used for the calculation is indicated, see Table 1.

In order to compare the 2HDM results with the corresponding MSSM values, we have systematically searched for the (allowed) regions across the MSSM parameter space where the optimal values for the cross-sections are attained. Besides the exception triggered by the resonant process $e^{+} e^{-} \rightarrow h^{0} h^{0} A^{0}$, whose maximum cross-section reads $\sigma(\sqrt{s}=1 \mathrm{TeV}) \sim 10^{-3} \mathrm{pb}$, the remaining $\sigma(3 H)$ are very small, namely they may reach $\sigma(\sqrt{s}=1 \mathrm{TeV}) \sim 10^{-6} \mathrm{pb}$ at most. We can thus assert that most of the $3 \mathrm{H}$ cross-sections in the MSSM are really tiny and, hence, very difficult to detect in practice. Let us finally stress that the extremely clean environment of the ILC should allow a relatively comfortable tagging of the three Higgs boson events for the typical $3 \mathrm{H}$ cross-sections that we have obtained in the general 2HDM case.

\section{Discussion and conclusions}

We have computed the leading-order cross-sections for triple Higgs boson production in a $e^{+} e^{-}$linear collider - cf. Eq. (1.1) - within the general 2HDM. We have shown that the production cross-sections may comfortably reach $0.1 \mathrm{pb}$ within type I $2 \mathrm{HDM}$. Such optimal rates are achieved for sufficiently large (or small) values of $\tan \beta(\tan \beta \gtrsim 20, \tan \beta<0.1)$. Moreover, in certain regions of the parameter space, and for the most favorable processes (such as $e^{+} e^{-} \rightarrow H^{+} H^{-} h^{0}$ ) the cross-sections can be pushed up to $\sim 1 \mathrm{pb}$. In spite of the fact that $3 \mathrm{H}$ production does not involve any kind of Higgs-fermion interaction, and hence the predicted rates should not depend on whether type I or type II $2 \mathrm{HDM}$ is considered, low-energy $B$-meson physics puts stringent constraints on $M_{H^{ \pm}}$in the type II case. Such scenarios with relatively heavy Higgs boson masses render maximum cross-sections that are roughly 10 times smaller, i.e. of order of $0.01 \mathrm{pb}$. The smaller number of events falls nonetheless in the range of $10^{3}$ per $100 \mathrm{fb}^{-1}$ of integrated luminosity. 
Remarkably, for both type I and type II 2HDM models the maximum cross-sections lie far above their MSSM counterparts (which typically remain at the tiny level of $\sigma \sim 10^{-6} \mathrm{pb}$ ).

Worth stressing is also the fact that, for the general $2 \mathrm{HDM}$, the maximum $3 \mathrm{H}$ production rates are fully comparable, and even larger (for type I models), than the optimal values achieved by the $2 \mathrm{H}$ processes $(\sigma \sim 0.1 \mathrm{pb})$. Such a result can be traced back to the potential enhancement of the Higgs boson self-interactions $(H H H)$ within the 2HDM, which cannot be realized in the MSSM case because of the SUSY invariance of the interactions. We conclude that the $3 \mathrm{H}$ final states in the general 2HDM can be a competitive, if not the dominant, Higgs boson production mechanism at the ILC. Owing to the extremely clean ILC environment, we expect that the leading $3 \mathrm{H}$ signatures could hardly be missed. The latter could be quite spectacular since they should reveal in the form of 6 heavy-quark jet final states. If a few, well identified, events of this kind would be detected, it should hint strongly at (non-SUSY) Higgs boson physics beyond the SM

Acknowledgments GF thanks an ESR position of the European network RTN MRTN-CT-2006035505 Heptools, and the hospitality of the HEP group at the Dept. ECM of the Univ. de Barcelona. DLV has been supported by the MEC FPU grant Ref. AP2006-00357; JG and JS in part by MEC and FEDER under project FPA2007-66665 and also by DURSI Generalitat de Catalunya under project 2005SGR00564. JG is thankful to the Universidad de Zaragoza, for their kind hospitality.

\section{References}

[1] A. Djouadi, H.E. Haber and P.M. Zerwas, Zeitsch. f. Physik C57 (1993) 569; A. Djouadi, H.E. Haber and P.M. Zerwas, Phys. Lett. B375 (1996) 203 ; A. Djouadi, V. Driesen, W. Hollik and J. Rosiek, Nucl. Phys. B491 (1997) 68; J.L. Feng and T. Moroi, Phys. Rev. D56 (1997) 5962.

[2] V. Driesen, W. Hollik and J. Rosiek, (1996); S. Heinemeyer, Int. J. of Mod. Phys. 21 (2006) 2659; A. Djouadi, W. Kilian, M. Muhlleitner and P.M. Zerwas, Eur. Phys. J C10 (1999) 45; T. Binoth, S. Karg, N. Kauer and R. Ruckl, Phys. Rev. D74 (2006) 113008.

[3] J. Guasch, W. Hollik, A. Kraft, Nucl. Phys. BB596 (2001) 66; J. Guasch, D. López-Val and J. Solà, work in progress.

[4] P. Osland and P.N. Pandita, Phys. Rev. D59 (1998) 055013; A. Djouadi, W. Kilian, M. Muhlleitner and P.M. Zerwas, Eur. Phys. J C10 (1999) 27; D.J. Miller and S. Moretti, Eur. Phys. J C13 (2000) 459; F. Boudjema and A. Semenov, Phys. Rev. D66 (2002) 095007.

[5] G. Ferrera, J. Guasch, D. López-Val and J. Solà, Phys. Lett. B659 (2008) 297.

[6] J.F. Gunion, H.E. Haber, G.L. Kane and S. Dawson, The Higgs hunter's guide, Addison-Wesley, Menlo-Park, 1990.

[7] S. Béjar, J. Guasch and J. Solà, Nucl. Phys. B600 (2001) 21; Nucl. Phys. B675 (2003) 270; [hep-ph/0101294]; S. Béjar, PhD Thesis, UAB, [hep-ph/0606138].

[8] M.B. Einhorn, D. R. T. Jones and M. J. G. Veltman, Nucl. Phys. B123 (1977) 89.

[9] P. Gambino and M. Misiak, Nucl. Phys. B611 (2001) 338; M. Ciuchini, G. Degrassi, P. Gambino, and G. F. Giudice, Nucl. Phys. B527 (1998) 21; F. M. Borzumati and C. Greub, Phys. Rev. D58 (1998) 074004 .

[10] T. Hahn, FeynArts 3.2, FormCalc and LoopTools user's guides, available from http: //www. feynarts. de; T. Hahn, Comput. Phys. Commun. 168 (2005) 78. 